Revue des patrimoines

\title{
Imagerie numérique et patrimoine culturel : enjeux scientifiques et opérationnels
}

Éditorial

Digital imaging and cultural heritage: scientific and operational issues

\section{Pascal Liévaux et Livio De Luca}

\section{OpenEdition Journals}

Édition électronique

URL : http://journals.openedition.org/insitu/22411

DOI : 10.4000/insitu.22411

ISSN : $1630-7305$

Éditeur

Ministère de la Culture

Référence électronique

Pascal Liévaux et Livio De Luca, «Imagerie numérique et patrimoine culturel : enjeux scientifiques et opérationnels », In Situ [En ligne], 39 | 2019, mis en ligne le 09 juillet 2019, consulté le 24 septembre 2020. URL : http://journals.openedition.org/insitu/22411 ; DOI : https://doi.org/10.4000/insitu.22411

Ce document a été généré automatiquement le 24 septembre 2020.

\section{c) $(1) \odot$}

In Situ Revues des patrimoines est mis à disposition selon les termes de la licence Creative Commons Attribution - Pas d'Utilisation Commerciale - Pas de Modification 4.0 International. 


\title{
Imagerie numérique et patrimoine culturel : enjeux scientifiques et opérationnels
}

\author{
Éditorial
}

Digital imaging and cultural heritage: scientific and operational issues

\author{
Pascal Liévaux et Livio De Luca
}

1 Depuis un demi-siècle, sciences et technologies du numérique n'ont cessé de progresser de concert, ouvrant une nouvelle ère pour la connaissance, la conservation et la diffusion des œuvres et des objets patrimoniaux. Tout d'abord par la recherche, puis, progressivement, dans le cadre de projets culturels construits dans le cadre de coopérations entre institutions patrimoniales et opérateurs professionnels mettant en œuvre des méthodes innovantes d'acquisition, d'observation et d'analyse multidimensionnelle. Des dispositifs de médiation inédits ont ainsi été inventés, expérimentés et évalués.

2 Tout d'abord réservé à des opérations exceptionnelles, l'usage de l'imagerie numérique s'est largement répandu à la faveur d'un processus de «démocratisation » stimulé par la simplification et l'automatisation progressive de l'instrumentation et des traitements. Par sa triple capacité à enregistrer le réel, à en expliciter les propriétés optiques et géométriques et à restituer les résultats de l'interprétation que l'on peut en faire, l'imagerie numérique, en deux ou en trois dimensions, s'impose aujourd'hui comme un outil de travail irremplaçable. 
Figure 1

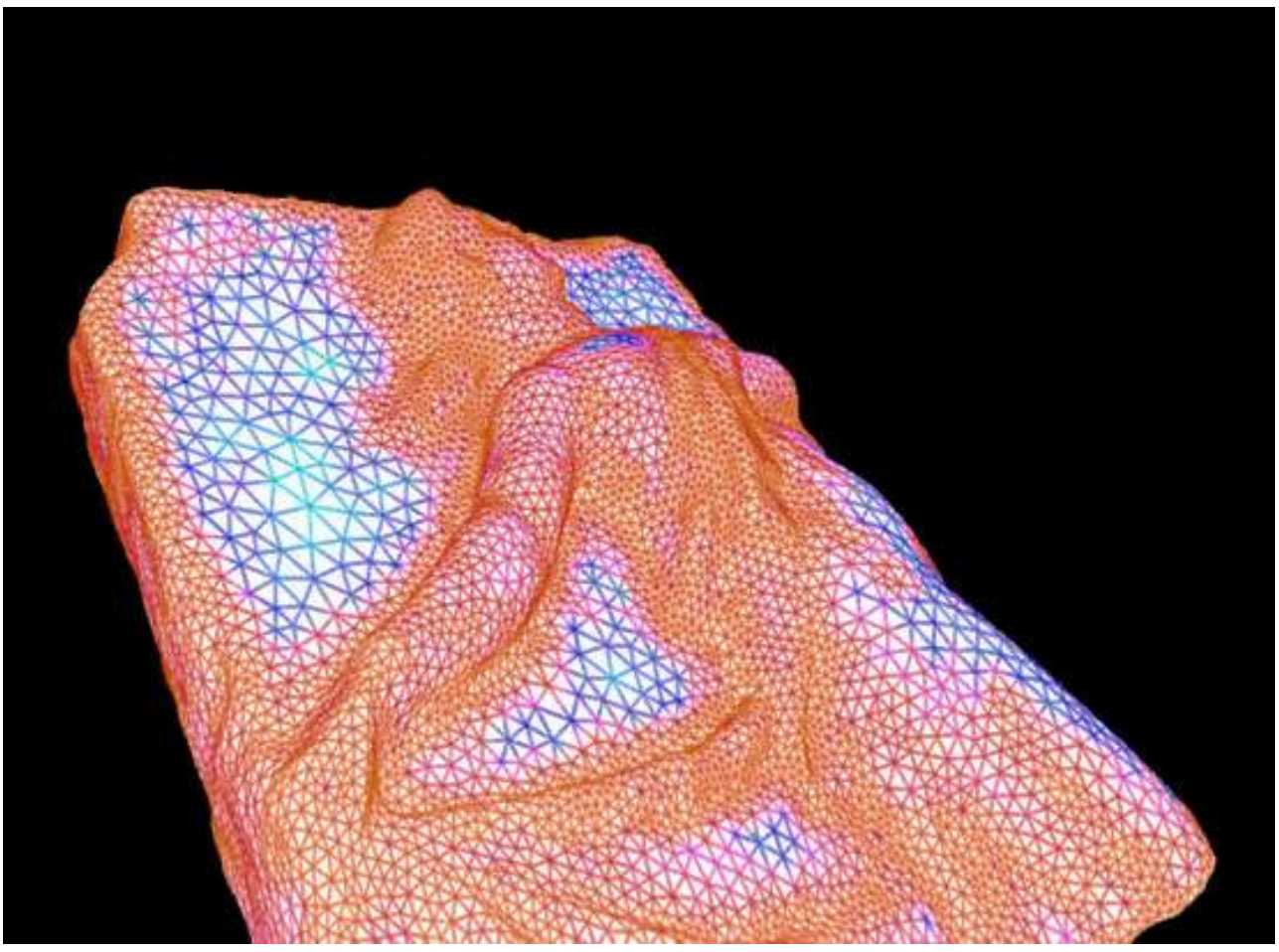

Mur de scène du théâtre antique d'Orange (Vaucluse). Fragment figurant un satyre dansant : carte des degrés d'accident.

Titien Bartette-ISCD. ( ) ISCD.

C'est ainsi que la panoplie d'instruments d'investigation et de diagnostic mobilisés pour l'acquisition de connaissances préalable aux restaurations s'enrichit jour après jour de capteurs et d'algorithmes d'analyse et de traitement de l'image. Dans le même temps, des usages innovants de l'imagerie numérique commencent à émerger dans le milieu des professionnels du patrimoine, à l'initiative de restaurateurs, de conservateurs, d'archéologues. Les responsables de sites et de collections font désormais appel à des sociétés prestataires de services maitrisant les technologies de numérisation, de traitement et de visualisation interactive, afin d'élaborer des circuits de visite, des préparations à la visite, des reconstitutions/restitutions d'œuvres, d'objets, de sites, des dispositifs interactifs, etc.

Dans les domaines de l'histoire de l'art et de l'archéologie, l'imagerie 3D, un temps surmédiatisée et parfois gadgétisée, permet notamment la restitution d'états antérieurs documentés ou hypothétiques, de différentes phases de la création. Elle est de plus en plus abordée dans un cadre scientifique faisant dialoguer sciences humaines et sociales, sciences expérimentales et sciences du numérique. C'est dans une telle approche interdisciplinaire que s'exprime au mieux son haut potentiel en termes d'organisation et de gestion de la documentation produite autour d'un objet patrimonial (étude historique, état de conservation, suivi temporel, etc.). La mémorisation et le partage des contenus numériques offrent la possibilité d'une véritable mise en commun des données élaborées par les différents spécialistes et services en charge de l'objet patrimonial, faisant de sa représentation numérique un point d'accès privilégié à la documentation photographique, bibliographique, analytique, archivistique, administrative, le concernant, accumulée au fil du temps et des opérations réalisées. 
Figure 2
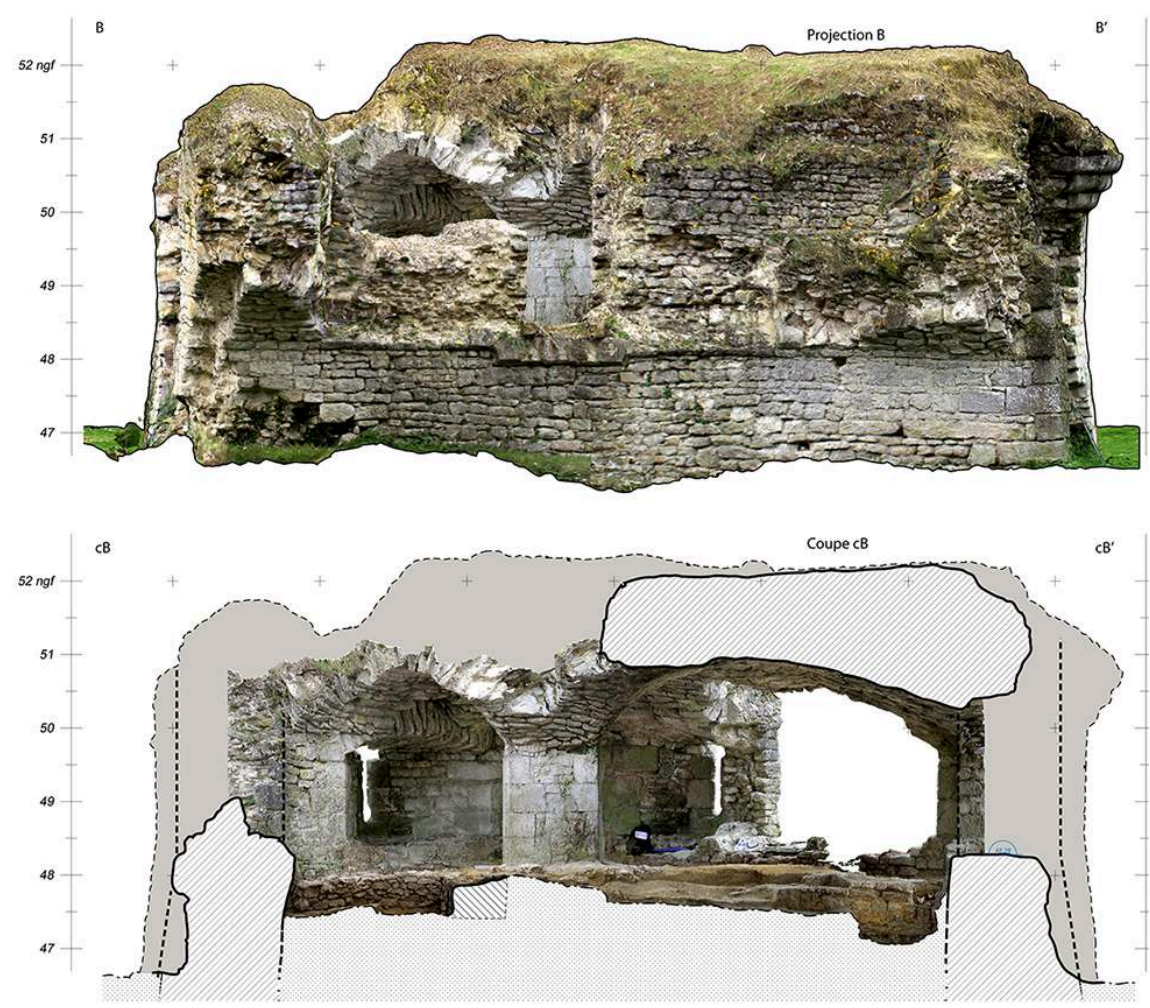

Orthophotographie, coupe longitudinale dans le ravelin du château de Beynes (Yvelines). F. Duban laisse en blanc les parties coupées et détaille les arrachements; ici les maçonneries coupées sont hachurées et les arrachements grisés.

(c) P. Raymond, L. Decock, 2016, Inrap.

5 En observant ce nouveau contexte de production de données et de connaissances, on comprend à quel point l'usage au quotidien de l'imagerie numérique fait apparaitre des enjeux et objets de recherche qui questionnent les différents acteurs sur leurs pratiques professionnelles aussi bien que sur le rapport qu'ils entretiennent avec les œuvres. C'est en effet bien au-delà des seuls défis techniques que les usages innovants du numérique pour l'étude, la conservation et la diffusion des patrimoines se construisent. Ils induisent de nouveaux modes d'observation, d'analyse et de représentation forcément conditionnés par la singularité des regards portés sur l'objet patrimonial et sur les connaissances mobilisées pour sa compréhension.

Ce bouleversement des pratiques stimule de nouveaux débats, fait apparaître de nouvelles préoccupations, de nouveaux enjeux. Il entraîne un processus d'appropriation aboutissant à la construction d'une véritable culture technologique intimement liée aux mécanismes de compréhension et d'interprétation. Il s'agit donc d'un double regard porté à la fois vers le futur pour anticiper l'émergence de nouveaux questionnements générés par l'usage du numérique (ainsi que par le cumul de contenus), et vers le passé pour souligner, réaffirmer et reconquérir la continuité historique des modes opératoires.

7 La richesse et la variété des contributions sélectionnées dans ce numéro à partir d'un grand nombre de propositions démontre s'il le fallait à quel point ce domaine, encore émergent, offre un environnement favorable à la réalisation de travaux associant 
chercheurs académiques et professionnels du patrimoine au sein de programmes allant de la recherche la plus fondamentale à la plus appliquée. Dans le cadre de ces travaux se déploient des efforts considérables d'harmonisation et d'intégration de compétences, de regards et de sensibilités diverses, dans un cadre pluridisciplinaire et interdisciplinaire rendant possible la construction collective d'approches innovantes. C'est la raison pour laquelle la revue In Situ a souhaité consacrer deux numéros à cette thématique. Tout en ne prétendant pas dresser un panorama exhaustif de la recherche et des développements technologiques dans le domaine de l'imagerie numérique appliquée au patrimoine, le premier esquisse un portrait, que nous espérons fidèle, d'une communauté dynamique, proposant et expérimentant des approches et des outils innovants, interrogeant sans cesse ses propres modes opératoires pour les renouveler par l'intégration de connaissances et compétences de plus en plus riches et variées.

Les articles sélectionnés portent sur des objets patrimoniaux très différents, monuments historiques, sites archéologiques et ensembles urbains, sculptures monumentales, art pariétal, cartes, objets de toutes tailles, etc., réalisés avec les techniques et les matériaux les plus divers, appartenant aux époques les plus variées. $\mathrm{Du}$ fait de leur grand nombre, ils ont été répartis en deux volumes constituant deux numéros en relation à deux grands champs d'application de l'imagerie numérique : pour l'observation et l'analyse du patrimoine dans le premier volume; pour la représentation et la transmission des connaissances dans le second.

Figure 3

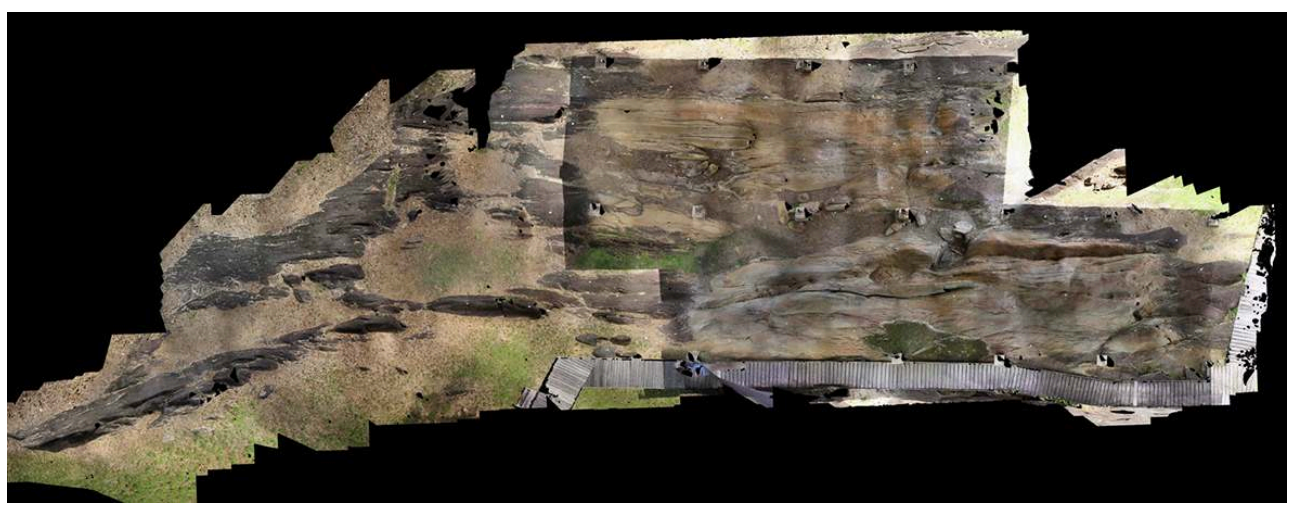

Ortho-photographie des roches gravées de la Carapa, Kourou (Guyane).

Phot. Franck Vautier. (c) Franck Vautier.

9 À l'intérieur de ce premier volume, on peut distinguer deux grandes catégories d'articles, la première intéressant le renouvellement technologique et méthodologique induit par les nouveaux moyens d'observation et d'enregistrement du réel, la seconde se penchant plus particulièrement sur l'impact du numérique dans la construction de nouveaux modes d'analyse, d'interprétation ou de conservation-restauration. Nous remercions vivement les contributeurs ainsi que l'équipe d'In Situ, particulièrement Françoise Cosler et Catherine Gros, d'en avoir rendu possible la publication dans les meilleures conditions. 


\section{AUTEURS}

\section{PASCAL LIÉVAUX}

Chef du département du Pilotage de la recherche et de la Politique scientifique, direction générale des Patrimoines, ministère de la Culture pascal.lievaux@culture.gouv.fr

\section{LIVIO DE LUCA}

Directeur de recherche au CNRS, directeur de l'UMR CNRS/MCC MAP (Modèles et simulations pour l'Architecture et le Patrimoine) livio.deluca@map.cnrs.fr 